\title{
At-Home Medical Equipment for the Physically Impaired using ZigBee and GPS Conrole
}

\author{
Anni Priyanka, Allu Venkateswara Rao, Dharma Raj Cheruku
}

\begin{abstract}
In the absence of anyone, old people and people who depend on others may fall down accidentally due to lack of strength. Durable Medical Equipment (DME) is medical equipment installed at home. The proposed system helps in activating and sending the DME to the nearby hospital which can save a patient's life. This system has two sections. One is the multiple Zigbee transmitting section installed at home which includes the DME and sensors and the other section is the Zigbee receiver present at the hospitals. Also, this system is independent of current-infrastructure such as cell-towers, internet etc. In case of any power outage for many days during any natural disaster, the patient status is sent through Zigbee protocol. The proposed system is designed by using ARM 32-bit microcontroller. This system includes GPS, ZIGBEE, temperature sensor and heart beat sensor modules. All these modules are attached to the target patient at home. The GPS finds out the location of the patient. The heart beat sensor gives the output in digital form when we kept the finger on the sensor. The room temperature was sensed by the temperature sensor. Once the controller gets the information from these devices, the information is sent over the Zigbee protocol to the Zigbee receiver section and allows the nearby hospital staff know about the condition and location of patient.
\end{abstract}

Keywords : GPS, DME, ZigBee, LM35 sensor, Heartbeat Sensor, LCD.

\section{INTRODUCTION}

For living in better way we use DME at home. Keeping this equipment in home also helps in completing daily activities. Some of the equipment includes walkers, oxygen tanks, nebulizers, CPCP, hospital beds and wheel chair etc. Medical devices can be active or static. Static devices are simple having few or no moving parts. While the active devices are bit complex. They require artificial power source such as a batter or electrical supply. These appliances perform more complex functions than static devices.

But, in case of power outage for many days during any natural disaster, the patient may face a life-threatening situation as they run on power. Though the DME devices are set-up with integrated batteries, they can with stand for more than one hour with lead batteries and with lithium batteries they can with stand up to more than three hours.. Also, the system should not rely on the electricity, infrastructure such as cell towers. Reporting here is a DME tracking system the

Revised Manuscript Received on December 13, 2019.

* Correspondence Author

A.Priyanka*, M Tech Student, ECE Department, Sri Sivani Institute of Technology, Chilakapalem, Srikakulam, Andhra Pradesh, India.

Allu Venkateswarar Rao, Assistant Professor, ECE Department, Sri Sivani college of Engineering, Chilakapalem, Srikakulam, Andhra Pradesh, India.

Prof. C. DharmaRaj, Dept. of EECE, GIT, GITAM (deemed to be University), Visakhapatnam. India
Zigbee protocol for the transmission of the data from the DME machines to the hospital zone.

\section{RELATED WORK}

There are many health monitoring systems being implemented for ICU patients. These systems are wired everywhere. Regular monitoring cannot be done to those patients when they are discharged. Also, there are some systems implemented wirelessly but the continuous monitoring of patient is not done. Those system monitors periodically.

Some health monitoring systems used the Mobile phone network and developed apps on it for convenient use. But, during the natural disasters, there may be power off. And, the mobile signals may also be unavailable because of tower destruction or any other issues for many days. At that point of time, those systems cannot be useful as they depend on the network in that area resulting in failing to update patient data to the hospitals.

So, the requirement for continuously monitoring the patient data and also sending the collected data from the controller to the nearby hospital during power outage on a ZigBee network which does not require any infrastructure like cell tower, power etc is done by using an ARM processor.

\section{PROPOSED SYSTEM FOR IMPLIMENTATION}

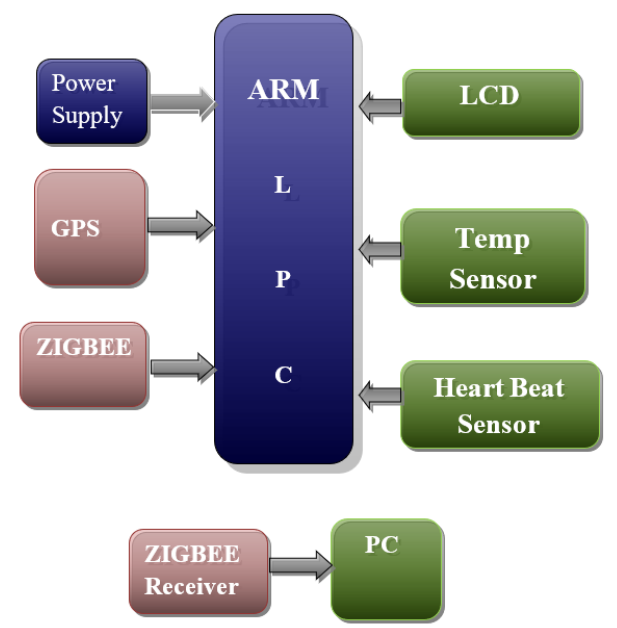

Fig1: Diagram for implementing the proposed system

In the proposed system, the microcontroller used is ARM7 LPC 2148. The different units used are Power supply unit, GPS, ZigBee, LCD, Sensors for Heartbeat temperature. The GPS and Zigbee units are connected to the microcontroller through serial communication and the sensors that is heart beat and temperature are connected to the microcontroller. 
The data from these sensors along with the GPS location in form of co-ordinates are transmitted over the Zigbee protocol from the transmitter section to the Zigbee

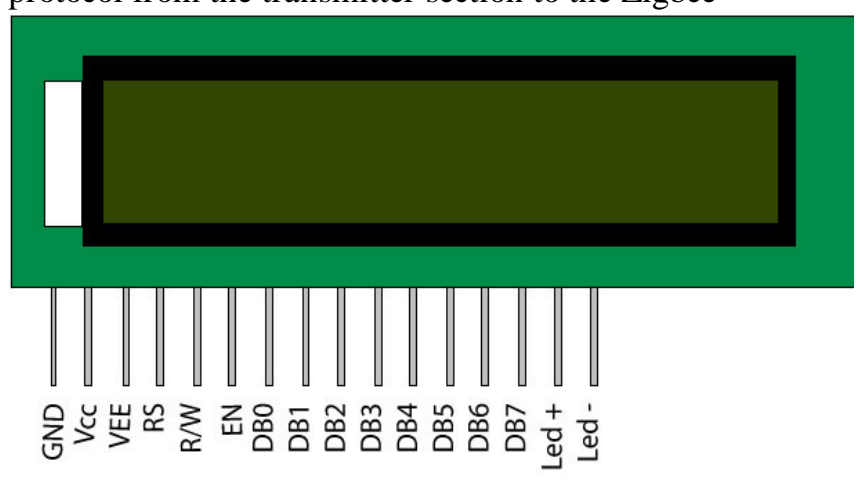

Fig 2: 16*2 LCD display

installed at the hospitals. In the power supply section for getting 5 volts we use a regulator 7805 .

In the proposed system, the doctors can monitor the patient data through wireless communication. The location of the patient is taken from the GPS module. In case of any power outage, the patient data is sent to hospital through the Zigbee protocol which is independent of the power, current infrastructure such a cell tower etc. which are unavailable during any natural disaster. And, the system uses the ARM7 LPC2148 microcontroller for controlling all the units.

\section{HARDWARE IMPLEMENTATION}

\subsection{ARM7 LPC2148 Microcontroller:}

LPC 2148 is a ARM7 controller with advanced 32 bit Reduced Instruction Set Computer (RISC) processor architecture developed by ARM holdings. LPC indicates low power low cost controller. This ARM7 microcontroller is designed with several in-built features and peripherals. Hence it will make more efficient as well as more reliable option for an application developer. Because of low power consumption and very small size, it is best for applications where miniaturization is a key requirement.

Features of LPC2148:

- $\quad$ LPC 2148 is a ARM7 controller( either 16 bit or 32 bit ).

- It gives changeable analog output with 10 bit digital to analog converter.

- Has 8000bit to 40000 bit on chip static Random access memory.

- $\quad 32000$ bit to 512000bit on chip memory.

- It has $32000 \mathrm{~Hz}$ clock input and Low power Real time clock.

- It has a watch dog pulse width modulated input.

- It interfaces with $0.4 \mathrm{Mbps}$ speed to UARTs and I2C-buses.

\subsection{ZigBee}

ZigBee operates with a specification of IEEE 802.15.4. It is a low-power, low-cost, wireless mesh network standard. ZigBee is used to create a network require a low data rate transfer, secure networking and energy efficient. It is designed to use low power digital radio signals for PAN and is an open global standard for wireless technology. The ZigBee networks operated over the frequencies $0.9 \mathrm{GHz}$ and
2.4GHz. The ZigBee specifications are updated and maintained by the IEEE 802.15 .4 standard.

\subsection{Liquid Crystal Display (LCD):}

It is a display with horizontal panel uses liquid crystals as its main shape of operation. LCDs are frequently developed in laptops, computer monitors, TVs, smart phones. LCDs set aside the display to be as thin as the cathode ray tube (CRT) technology. It utilizes very few quantity of power than Light emitting diodes as they work on the principle of blocking light rather than the light emitting. The flow of liquid crystal in an LCD makes an image on the screen. Now a day's LCDs have certain thin film transistors for obtain better results The thin film transistors include capacitors which enable individual pixels to actively retain their charge.

\subsection{Temperature Sensor:}

LM35 is a temperaturesensor which measures analog output voltage proportional to the given temperature. The output voltageprovided by the temperature sensor is in ${ }^{0} \mathrm{C}$. The sensitivity of $\mathrm{LM} 35$ is $10 \mathrm{mV} /{ }^{0} \mathrm{C}$. It doesnot require any external calibration circuitry. It is a 3 -terminal device. It is used to determine the surrounding temperature ranging from $-55^{\circ} \mathrm{C}$ to $150^{\circ} \mathrm{C}$. The output obtained by this sensor is more precise than the out put obtained bythermistor.

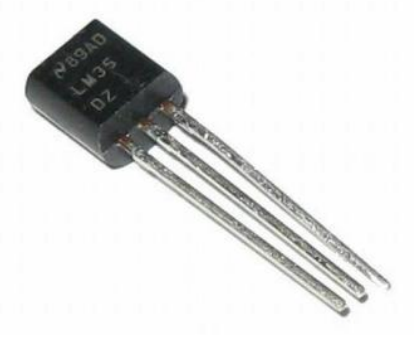

Fig 3: LM35 Temperature Sensor

\subsection{GPS:}

GPS is used to send and receive radio frequency signals which are navigated by the satellite Based station. We can use this technology while driving, flying, running etc. Using this technology, we can get the nearby location of any shopping mall, cinema theaters etc, when we are out of town.

\subsection{Power Supply:}

The power supply unit consisting of rectifier converts AC to pulsating DC supply. Regulator is a device which converts pulsating DC to constant DC supply. Each of the blocks is described in more detail below:

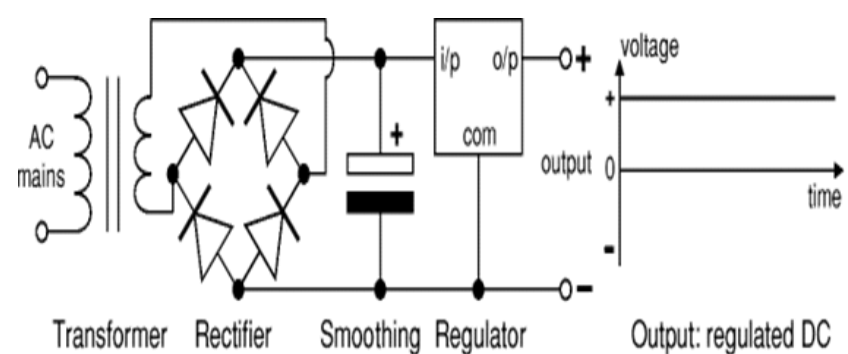

Fig 4: Regulated DC supply Unit 
- $230 \mathrm{v}$ Ac supply is given to the step down transformer to step down high voltage AC to low voltage AC supply.

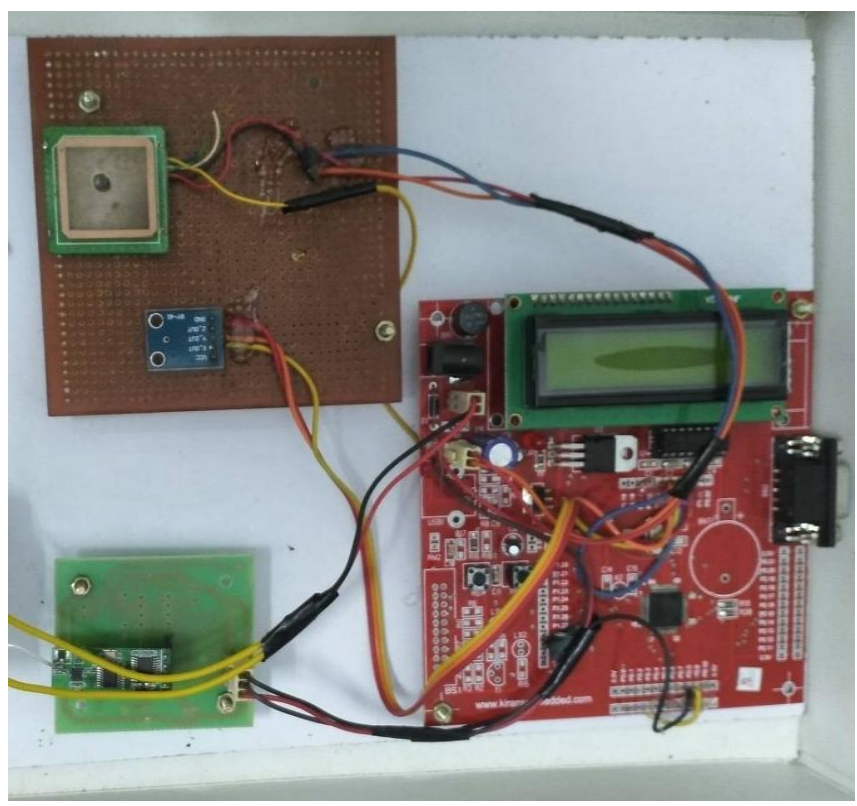

Fig 5

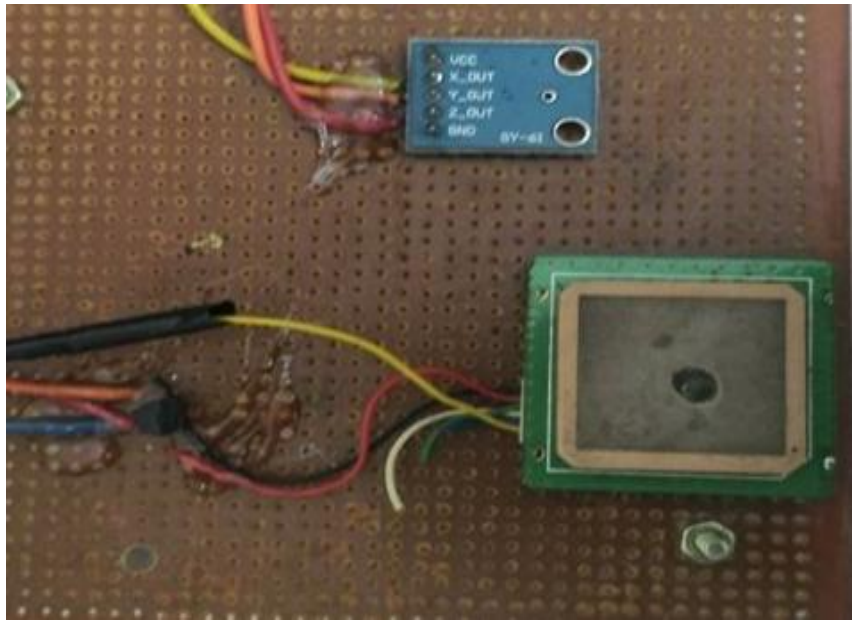

Fig 6 AC to pulsating DC

- Filters are used to remove rippels present in the DC and provide pure DC.

- Three terminal voltage Regulator used to get constant DC output.

\section{- 4.7. Heart Beat Sensor:}

- This sensor measures the rate (speed) of heart. Heartbeat is the echo of valves in human being heart. The rate of heart beat is measured by the number of times the heart beats per minute. It works on the principle of Photo phlethysmography. By this principle, the amount of change in the flow of blood volume in an organ is calculated by the intensity of the light is absorbed by the blood. Mostly, the light source in a heartbeat sensor will be an Infra Red device and the detector for light will be a photo Diode, a Photo Transistor or a light dependent resistor. Usually for athletes and patients monitoring the rate of heart is crucial as it determines the heart condition.

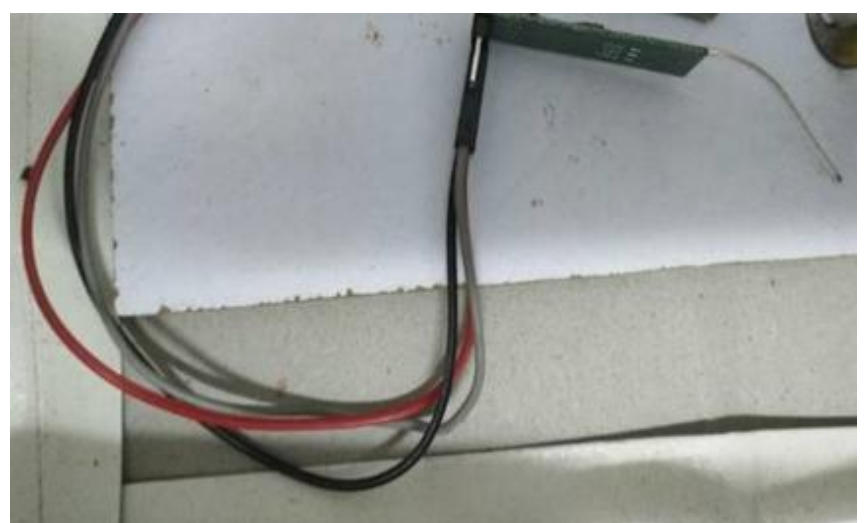

Fig 7: Receiver Zigbee Section connected through USB

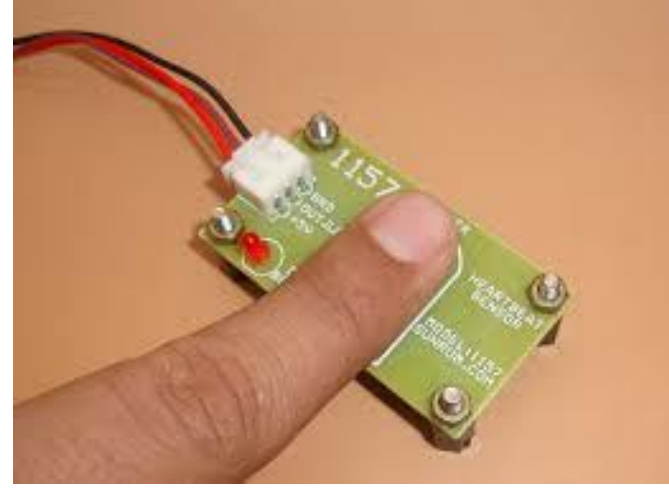

Fig 8: Heartbeat Sensor

\section{RESULT}

When the power is off, all the collected data from GPS, temperature sensor and heart beat sensor is sent through ZigBee protocol from the transmitting At-Home section to the receiver ZigBee at the nearby hospitals. Also, the data from the GPS information regarding its co-ordinates and the data from the temperature sensor will be displayed on the LCD display

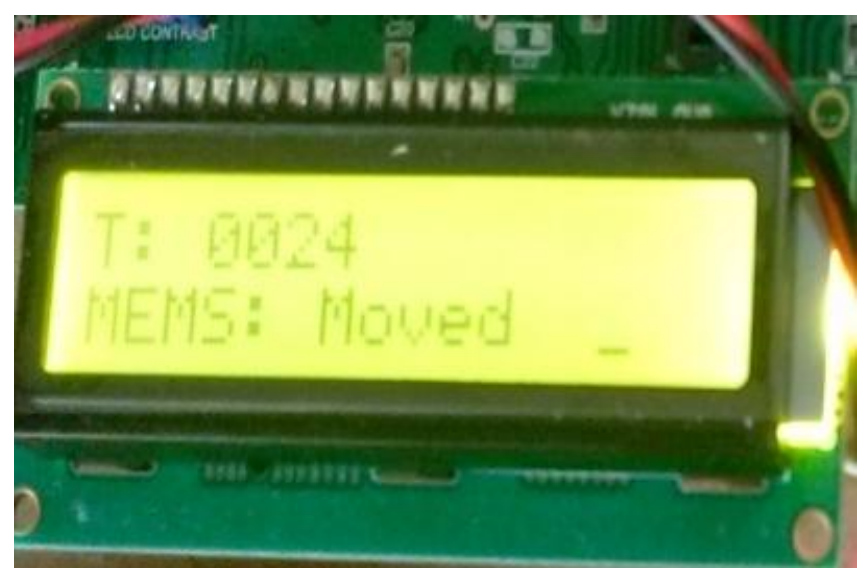

Fig 9: Output for test data

\section{CONCLUSION AND FUTURE SCOPE}

By giving some test data, the designed system's requirement for transmission of patient location information, status of DME system to the nearby hospitals in case of power outage during any natural disaster is successfully done and tested. The patient can get help from the doctor in case of any emergency successfully even

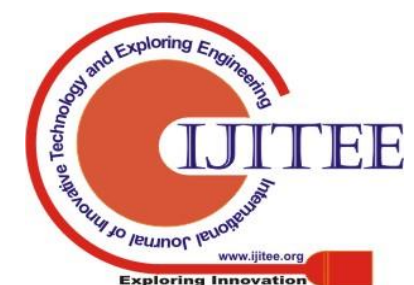


when they are distant from each other as this proposed system acts like communication link between them with the patient health data and location. Multiple parameters like blood pressure, age, weight etc. can be incorporated for scheming in future. Also with GPRS unit, the range of the data transmission can be increased only if there is availability of internet.

\section{REFERENCES}

1. Priya.u , Thakare, "smart assistance system for the visually impaired". International journal of scientific and research publications, 7:12 (2017)

2. Guojin Li, Jing, "Application of the medical care system based on ZigBee technology", Electrical and Electronics Engineering, IEEE Symposium (2012).

3. G. Balakrishna, G. Sainarayana, "A stereo image processing system for visually impired".

4. Durable medical equipment http://en.wikipedia.org/wiki/Durable_medical_equipment.

5. Prof. Priya U,Thakare, Kote Shubam,etl; "Smart assistance system for visually Impaired, International journal of scientific and research publications, vol:7, issue 12 (2017).

\section{AUTHORS PROFILE}

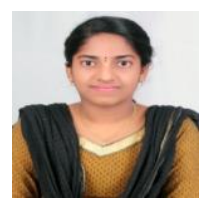

Anni Priyanka, M Tech scholar, ECE Department, Sri Sivani Institute of Technology, Chilakapalem, Srikakulam, Andhra Pradesh.

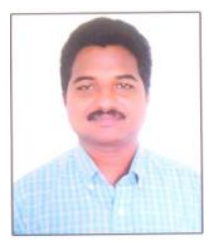

Allu Venkateswara Rao,presently Working as Assistant Professor in ECE Department, Sri Sivani college of Engineering, Chilakapalem, Srikakulam, Andhra Pradesh, INDIA.532402. He received his M.Tech from JNTU Hyderabad in 2008 and 2010. He is pursuing his Ph.D from GITAM university, Visakhapatnam .He published 8 Journal.

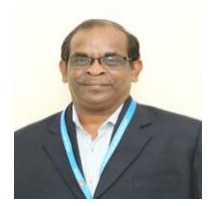

Dharma Raj Cheruku, Prof. C. Dharma Raj is currently working as professor in ECE department and in-charge principal, GITAM Institute of technology (GIT), GITAM Visakhapatnam, He received his B.E from Andhra university and M.E from Osmania university. He received his Ph.D from GITAM university in 2012. He worked as scientist "B" in LRDE, Bangalore. He served many positions in GITAM for the past 29 years. He had published 29 papers in reputed journals like springer, IEEE etc. He wrote 7 text books in E.C.E and guided $10 \mathrm{Ph} . \mathrm{D}$ students. His area of interest includes Electromagnetic field theory, Antennas, microwave and Engineering and wireless communications. 